\title{
PRACTICAL ONLINE CONDITION MONITORING OF GEARBOX OIL USING NON-DISPERSIVE INFRA-RED SENSORS
}

\author{
M.A.Parker*, A.Cleary ${ }^{\dagger}$, A.W.Hamilton \\ *University of Strathclyde, UK, max.parker@strath.ac.uk ${ }^{\dagger}$ University of Strathclyde, UK, alison.cleary@strath.ac.uk \\ *University of Strathclyde, UK, andrew.w.hamilton@strath.ac.uk
}

Keywords: Condition monitoring, wind turbine, gearbox oil, oil analysis, NDIR.

\begin{abstract}
A study has been carried out on the feasibility of using nondispersive infra-red (NDIR) analysis for online condition monitoring of the degradation of wind turbine gearbox oil. In particular, the effects of varying the size of the gap through which the oil flows was analysed, with considerably larger gaps of $1-3 \mathrm{~mm}$ used than reported elsewhere. Due to the high viscosity of gearbox oil, a large gap size is desirable for online monitoring. Old and new oil were found to produce a different output from the detector, suggesting that online condition monitoring of oil using NDIR is viable with large gap sizes, although the largest gap size produced some anomalous results.
\end{abstract}

\section{Introduction}

At present, the lubrication oil in wind turbine gearboxes is replaced in the turbine annual service, irrespective of the actual condition of the oil. If the turbine loading between oil changes is light, this means that the oil could be discarded while it still has considerable lifetime remaining. Conversely, if the turbine loading is heavy, the wear-reducing properties of the oil (such as the levels of extreme-pressure additives) could be reduced before it is replaced, resulting in wear to the gearbox and a reduction in gearbox lifetime [6].

Fourier-transform infra-red (FTIR) spectroscopy, which measures absorption of infra-red energy at different wavelengths, is one method of analysing the chemical composition of the oil, and hence its condition. Inline sampling, where continual monitoring occurs, has been demonstrated for particular fluids [10]. However gearbox oils have particularly high viscosities, preventing a number of methods from being applied. Lower cost systems, based on tunable Fabry-Perot spectrometers have been proposed [12], but are still have a significant cost and complexity. Nondispersive infra-red (NDIR) analysis concentrates on measuring the absorption at specific wavelengths relating to the chemical components of interest. Such equipment is considerably cheaper, and has been demonstrated with a variety of fluids $[1,3,9,11]$. Such a system could be incorporated in each turbine as part of an online oil condition monitoring package consisting of multiple low-cost sensors, for improved accuracy and diagnosis ability [h].

An overview of a typical test system used for gearbox oil NDIR analysis is shown in Figure 1. A sampling pump takes oil from the gearbox sump or oil reservoir, and pumps it through a test cell. This sampling loop is needed due to pressure and flow requirements. A thermal emitter shines broadband IR energy through a test sample, and a narrowband filter extracts the wavelength of interest, which is detected using a detector based on one of several principles. This system therefore measures the absorption at a specific wavelength determined by the narrowband filter. A complete measurement system will also measure the absorption at a control wavelength, to compare with that at the wavelength of interest, which helps minimise temperature effects. This can be achieved by either using a second test cell with a different narrowband filter, or by using a mechanical system to swap between two filters. Another option is to use a cluster of detectors with different narrowband filters, and a single emitter [9], but this requires a custom-made detector, which is more applicable for a production system.

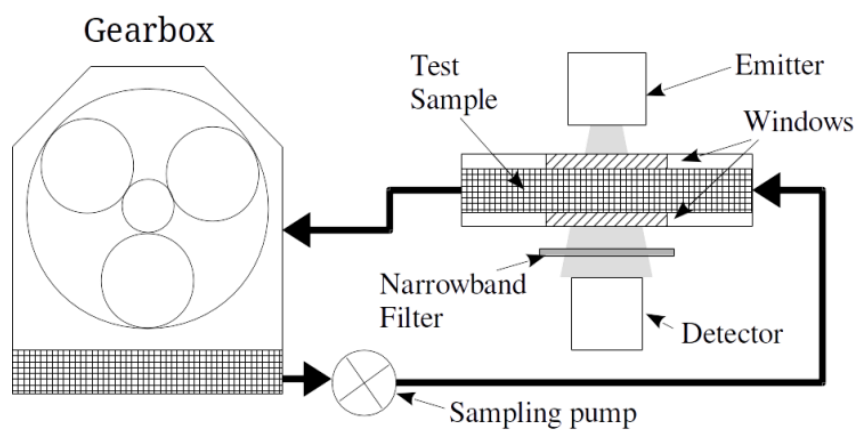

Figure 1 Gearbox NDIR-based online condition monitoring setup.

NDIR-based oil condition monitoring has been demonstrated, using both fixed samples of oil [1] and online systems in which oil is pumped through a test cell [9]. In all cases, the thickness of the test sample (i.e. the gap between the windows) is around $0.1-0.2 \mathrm{~mm}$. The relationship between the incident IR intensity $I_{0}$, and the transmitted intensity $I$, for an absorption path length $l$ is given by the Lambert-Beer equation[11]: 


$$
I=I_{0} e^{-k l c}
$$

Where $k$ is the extinction coefficient and $c$ the concentration of the IR active component. This means the detector output will decrease rapidly with increasing gap size, and a small size is desirable for maximum measurement sensitivity. A small gap size requires a high pressure in order for the oil to flow, requiring a high pressure pump and window materials able to withstand the pressure. This is especially the case for gearbox oil, which has a particularly high viscosity.

The aim of this research is to investigate the practicality of increasing the gap size, which would allow a cheaper low pressure sampling pump to be used, or for a sensor to be placed in the oil circulation path. The effect of increasing the gap size is to reduce the amplitude of the detector output signal, which will require a greater level of amplification, resulting in a significantly noisier signal. It will be shown that this can be partially mitigated by averaging the measured signal over a large number of samples. This is valid as the timescale for degradation, in the range of weeks or months, is much longer than the averaging period.

\section{Detection Principal}

Different oils can show different degradation mechanisms, leading to different changes in IR absorption spectrum. Many studies on the use of NDIR sensors for oil analysis concentrate on the analysis of engine oils. These tend to degrade through oxidation, which leads to increased absorption at wavenumbers from $1690 \mathrm{~cm}^{-1}$ to $1760 \mathrm{~cm}^{-1}$, due to formation of compounds containing the carbonyl $(\mathrm{C}=\mathrm{O})$ bond [1,3]. Phosphate ester hydraulic oils degrade through water absorption, which can react with the oil. This shows up in the region around $3500 \mathrm{~cm}^{-1}$ [9]. For gearbox oils, oxidation may be much more subtle, especially with high-performance synthetic oils, and condition monitoring is based on analysing the depletion of the oil additives [12], which can vary depending on the manufacturer and oil brand. For instance, in [12] the level of molybdenum in the gear oil, from the antiwear additive, is analysed using IR spectroscopy. The changes to the spectrum are subtle and are therefore unsuitable for NDIR analysis.

Samples of new and used synthetic wind turbine gear oil (MobilGear XMP320) were obtained, and analysed using FTIR analysis to find any differences in absorption spectrum [8]. By analysing the spectra of the oil when it is new and comparing it to spectra of old oil, suitable regions of interest can be identified for analysis [7]. The FTIR spectra are shown in Figure 2, where the most significant difference is a peak at a wavenumber of $1740 \mathrm{~cm}^{-1}$ which is present in new oil but not in used oil. This peak corresponds to a carbonyl bond, which can represent an ester-based additive [3]. Although the chemical composition of the oil is not known, and unlikely to be provided by the manufacturer, the fact that the peak disappears with oil age suggests that it represents an additive which is being depleted, and is therefore a good candidate for measurement. The used oil was around 4 years old, and was classified as degraded from other tests.

Narrowband IR filters were chosen with transmission wavenumbers of $1740 \mathrm{~cm}^{-1}$ and $2040 \mathrm{~cm}^{-1}$, the latter provides a reference signal in a region where the absorption does not vary significantly between new and used oil.

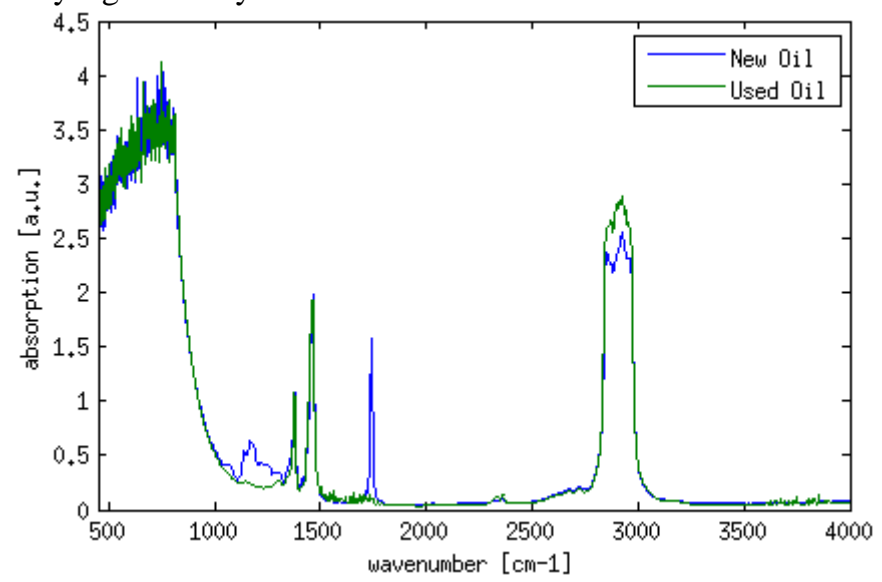

Figure 2 FTIR spectra of new and used oil

\section{Instrumentation Design}

The block diagram for the test system is shown in Figure 3. The emitters are micro-machined thermal emitters, with a power of $450 \mathrm{~mW}$, and are driven by a regulator which sets the emitter voltage based on a signal from a microcontroller. The microcontroller monitors the emitter current and sets the voltage to achieve a constant power output. This is necessary as the emitter resistance can vary between devices and over the lifetime of the emitter [2]. The outputs of the detectors are amplified and measured by the microcontroller, and the resulting output is logged by a PC.

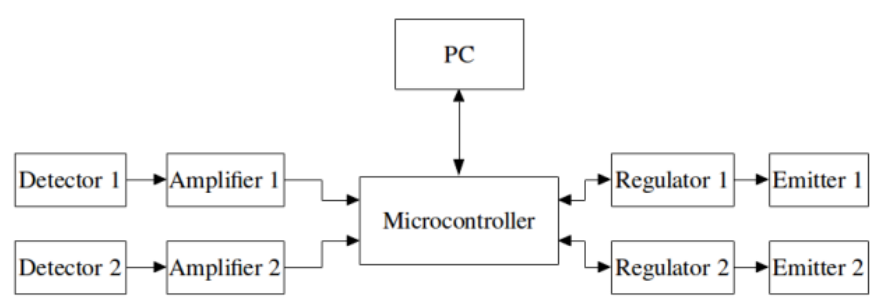

Figure 3 System block diagram

Two types of detectors can be used: thermopiles, based on the thermoelectric effect, and pyro-detectors, based on the pyroelectric effect. Both detect the IR intensity by measuring the heating of the detection element, but thermopiles produce a voltage output proportional to the incident IR energy while the output of pyro-detectors is related to the change in incident energy. While most of the studies cited in this paper use thermopiles for simplicity, pyro-detectors have a greater sensitivity. Testing with both types of detector showed that with larger gap sizes, thermopiles required an extremely high level of amplification, and the amplified signal was dominated by interference at the mains frequency even when operating the test system from batteries. For this reason, pyro- 
detectors were chosen. The chosen pyro-detector was the LHi807 TC G17 from Excelitas, specified with the G17 sapphire filter giving good transmission at wavelengths up to $6 \mu \mathrm{m}[4]$.

The emitter is switched on and off at a rate of $1 \mathrm{~Hz}$, and the amount by which the pyro-detector varies over this switching period depends on the strength of the IR energy received. A typical pyro-detector output signal is shown in Figure 4. The pyro-detector output is sampled at the ends of the emitter on and off periods, and for greater accuracy 32 samples are taken before each switching event in quick succession and averaged.

For the proof of concept presented here, only a single channel emitter and detector was implemented, with the narrowband filters swapped over manually to measure absorption at each wavelength.

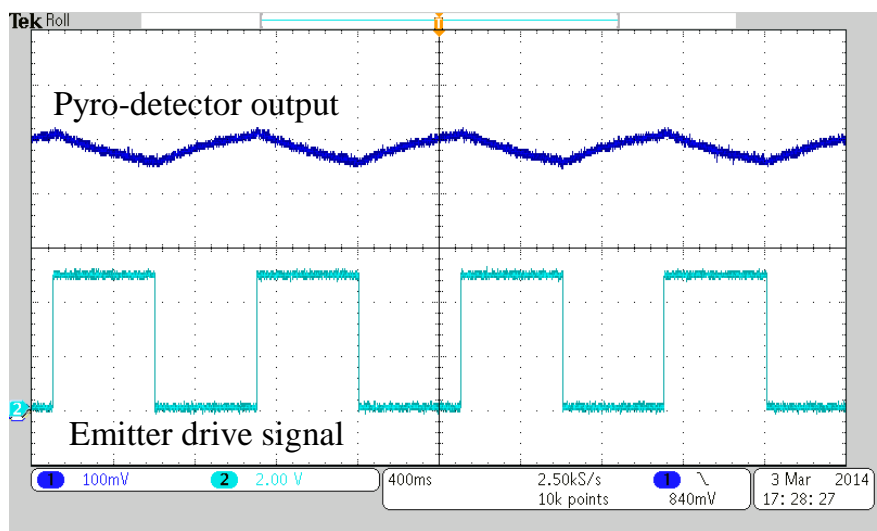

Figure 4 Typical pyro-detector output waveform

\section{Test Cell Design}

A simple test cell was constructed from aluminium, with top and bottom plates holding silicon windows, and $1 \mathrm{~mm}$ thick sandwich plates providing an oil channel. The thickness of the oil channel can be varied by changing the number of sandwich plates. A non-setting silicone gasket sealant was used between the plates. The components of the test cell are shown in Figure 5, and the assembled test cell shown in Figure 6. For each test, oil is introduced to one end of the test cell and allowed to flow through until the channel is filled.

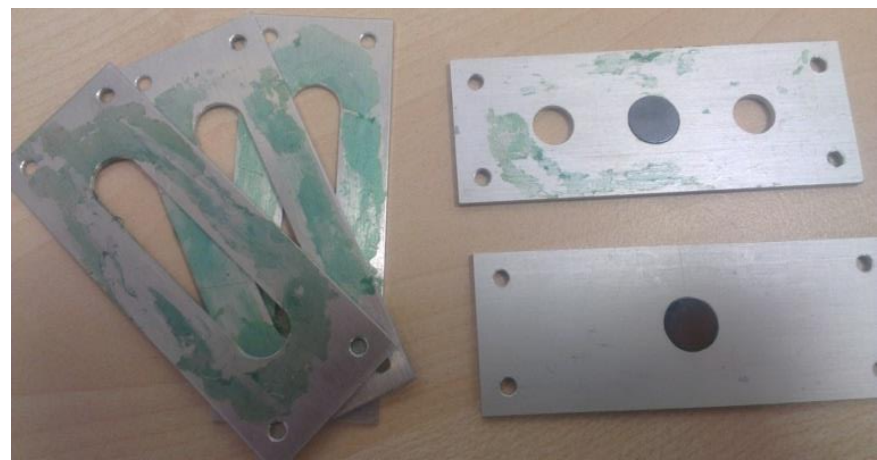

Figure 5 Test cell components
The detectors are soldered to a PCB (part of a prototype for a 2-channel unit), and the narrowband filters are attached to an aluminium carrier, which is shown in Figure 7. This shields the detectors from ambient light which could affect the detector output.

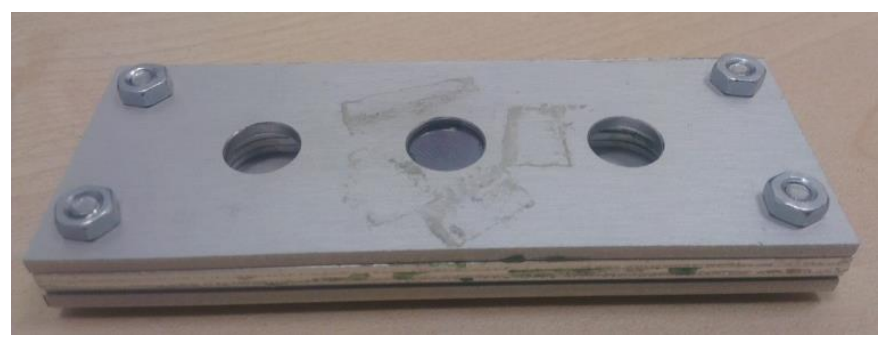

Figure 6 Test cell, assembled with 3mm gap

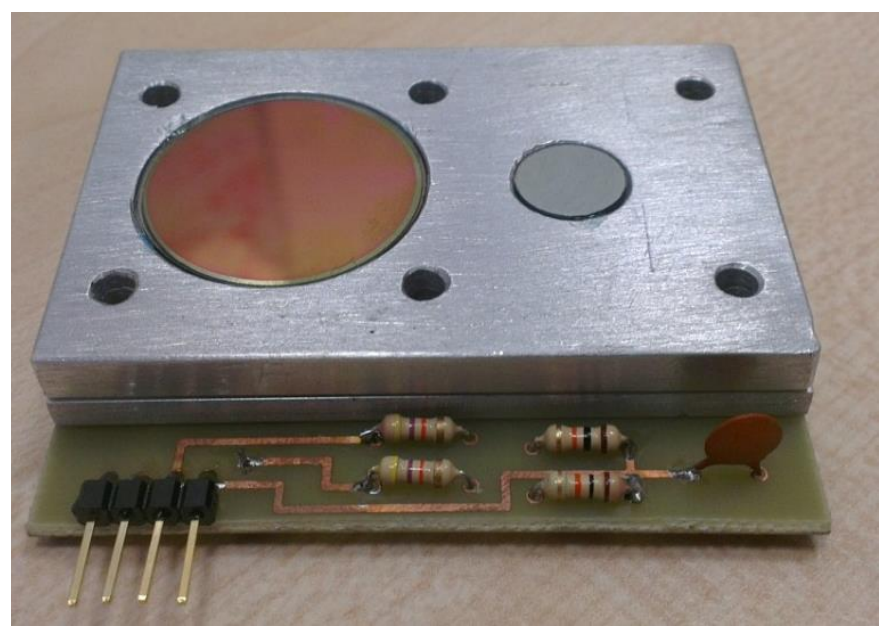

Figure 7 Detector PCB and narrowband filter holder

\section{Test Procedure, Results and Analysis}

For the testing, the test cell was assembled with the required number of spacer plates and placed in a stack with the emitter, detector and filters. When changing between old and new oil, the inside of the test cell was cleaned.

Initial testing was carried out with a fixed amplifier gain of 100 , and with the signal measured using an oscilloscope, in order to determine the required amplifier gains for logging with the microcontroller. For this test, the position of the detector and emitter relative to the top and bottom of the test cell were kept constant, but alignment was by eye, which could lead to inaccuracies in the results. The sensor output voltages are shown in Table 2.

For all gap sizes, the output voltage for the detector with $5.74 \mu \mathrm{m}$ filter was found to be a similar fraction of that with the $4.9 \mu \mathrm{m}$ filter. For new oil, the fraction was found to be lower, at around $1.4 \%$, compared with the new oil at around $6 \%$, as expected. Even with used oil, where absorption at $5.74 \mu \mathrm{m}$ should be equal to that at $4.9 \mu \mathrm{m}$, the detected signal is considerably lower. This is due to the $5.74 \mu \mathrm{m}$ filter having a narrower bandwidth and lower peak transmissivity. For the $3 \mathrm{~mm}$ gap with new oil, no signal was detectable. Assuming a 
ratio to the $4.9 \mu \mathrm{m}$ signal of $1.4 \%$, the signal output should be around $20 \mu \mathrm{V}$, which is only just above the typical noise level of the detector of $15 \mu \mathrm{V}$ [4], suggesting that it will not be possible to detect a signal over the noise.

\begin{tabular}{|l|l|l|l|l|}
\hline & \multicolumn{4}{|l|}{ Gap size (mm) } \\
\hline Oil & Filter & $\mathbf{1}$ & $\mathbf{2}$ & $\mathbf{3}$ \\
\hline \multirow{2}{*}{ Used } & $4.9 \mu \mathrm{m}$ & $15.6 \mathrm{mV}$ & $7.4 \mathrm{mV}$ & $2.08 \mathrm{mV}$ \\
XMP320 & $5.74 \mu \mathrm{m}$ & $1.04 \mathrm{mV}$ & $380 \mu \mathrm{V}$ & $120 \mu \mathrm{V}$ \\
& Ratio & $6.7 \%$ & $5.1 \%$ & $5.8 \%$ \\
\hline \multirow{2}{*}{ New } & $4.9 \mu \mathrm{m}$ & $20.6 \mathrm{mV}$ & $4.7 \mathrm{mV}$ & $1.44 \mathrm{mV}$ \\
XMP320 & $5.74 \mu \mathrm{m}$ & $300 \mu \mathrm{V}$ & $60 \mu \mathrm{V}$ & $0 \mathrm{~V}$ \\
& Ratio & $1.5 \%$ & $1.3 \%$ & $0 \%$ \\
\hline
\end{tabular}

Table 2 Sensor output voltages from initial testing

Amplifier gains were chosen based on the these results, and refined with further testing, to give a peak to peak input voltage to the microcontroller of between 0.5 and $3 \mathrm{~V}$ with used oil, with the signal varying around an average of $2.5 \mathrm{~V}$. The amplifier and detector were supplied with a single-rail $5 \mathrm{~V}$ supply from a linear regulator, in order to avoid potentially exceeding the voltage limits of the microcontroller analogue inputs. While the amplifier featured a rail-to-rail output, it was felt that the amplifier would have reduced performance near the supply rails so the signal amplitude was limited. The regulator also serves to isolate the detector power supply from fluctuations caused by the emitter switching, the emitter being fed from the main $10 \mathrm{~V}$ supply. The selected amplifier gains are given in Table 1, which are set by changing resistors within the amplifier circuit.

\begin{tabular}{|l|l|l|l|}
\hline Gap size & $\mathbf{1 m m}$ & $\mathbf{2 m m}$ & $\mathbf{3 m m}$ \\
\hline $\mathbf{4 . 9} \boldsymbol{\mu m}$ & 101 & 101 & 631 \\
\hline $\mathbf{5 . 7 4} \boldsymbol{\mu m}$ & 101 & 631 & 10,201 \\
\hline
\end{tabular}

Table 1 Selected amplifier gains

In these tests, the distance between the emitter and detector was kept constant with different gap sizes. As the IR intensity decreases with distance from the emitter in accordance with the inverse-square law, keeping the distance constant means that the change in detector output will only depend on the changing absorption by the oil in the gap. For these tests, the microcontroller records the detector voltage with the emitter on and off, and sends the data to a PC. As the emitter switches at $1 \mathrm{~Hz}$, the $\mathrm{PC}$ will receive a data point every second. The test is left to run for 10 minutes, giving around 600 data points, and the mean and standard deviation are calculated.

The mean sensor readings are shown in Table 3, which also shows the ratio between the $5.74 \mu \mathrm{m}$ and $4.9 \mu \mathrm{m}$ readings. For each oil age, the ratio is found to vary with gap size, unlike the previous experiment where it is constant. This is due to the gap between the emitter and detector being kept constant rather than varied with gap size. In all gap sizes, the ratio for new oil is significantly lower than for used oil, as predicted by the FTIR analysis. The ratio for new oil with a $3 \mathrm{~mm}$ gap is extremely low, and possibly an anomalous result - the $4.8 \mu \mathrm{V}$ sensor reading for the $5.74 \mu \mathrm{m}$ filter is significantly below the noise threshold of the detector.

The standard deviation of the sensor voltages is given in Table 4, and is generally significantly below the signal level, suggesting an accurate sampling of the sensor output. The standard deviations for 2 and $3 \mathrm{~mm}$ gaps for new oil with the $4.9 \mu \mathrm{m}$ filter are larger than the others, suggesting the presence of interference, but still small in comparison with the signal amplitude. For the $3 \mathrm{~mm}$ gap with new oil and the $5.74 \mu \mathrm{m}$ filter the standard deviation is $25 \%$ of the signal amplitude, but is significantly lower than the noise level of the detector and lower than the signal itself. This suggests that the combination of filtering within the amplifier and the multi-sampling carried out for each reading by the microcontroller is able to mitigate the detector noise. The implication of this is that the apparently anomalously low reading for this filter and gap size is due to some limitation in the detector or amplifier.

\begin{tabular}{|l|l|l|l|l|}
\hline & \multicolumn{4}{|l|}{ Gap size (mm) } \\
\hline Oil & Filter & $\mathbf{1}$ & $\mathbf{2}$ & $\mathbf{3}$ \\
\hline \multirow{2}{*}{ Used } & $\mathbf{4 . 9} \boldsymbol{\mu m}$ & $34.7 \mathrm{mV}$ & $11.4 \mathrm{mV}$ & $3.68 \mathrm{mV}$ \\
XMP320 & $\mathbf{5 . 7 4} \boldsymbol{\mu m}$ & $4.11 \mathrm{mV}$ & $0.802 \mathrm{mV}$ & $0.144 \mathrm{mV}$ \\
& Ratio & $11.8 \%$ & $7.05 \%$ & $3.91 \%$ \\
\hline \multirow{2}{*}{ New } & $\mathbf{4 . 9} \boldsymbol{\mu m}$ & $27.7 \mathrm{mV}$ & $6.42 \mathrm{mV}$ & $2.66 \mathrm{mV}$ \\
XMP320 & $\mathbf{5 . 7 4} \boldsymbol{\mu m}$ & $687 \mu \mathrm{V}$ & $97.7 \mu \mathrm{V}$ & $4.8 \mu \mathrm{V}$ \\
& Ratio & $2.48 \%$ & $1.52 \%$ & $0.18 \%$ \\
\hline
\end{tabular}

Table 3 Mean sensor output voltage from final testing

\begin{tabular}{|l|l|l|l|l|}
\hline & \multicolumn{4}{|l|}{ Gap size $(\mathbf{m m})$} \\
\hline Oil & Filter & $\mathbf{1}$ & $\mathbf{2}$ & $\mathbf{3}$ \\
\hline Used & $\mathbf{4 . 9} \boldsymbol{\mu m}$ & $58.3 \mu \mathrm{V}$ & $44.9 \mu \mathrm{V}$ & $15.3 \mu \mathrm{V}$ \\
\cline { 2 - 5 } XMP320 & $\mathbf{5 . 7 4} \boldsymbol{\mu m}$ & $38.3 \mu \mathrm{V}$ & $26.1 \mu \mathrm{V}$ & $6.9 \mu \mathrm{V}$ \\
\hline \multirow{2}{*}{ New } & $\mathbf{4 . 9} \boldsymbol{\mu m}$ & $44.9 \mu \mathrm{V}$ & $120.7 \mu \mathrm{V}$ & $74.6 \mu \mathrm{V}$ \\
\cline { 2 - 5 } XMP320 & $\mathbf{5 . 7 4} \mu \mathrm{m}$ & $41.6 \mu \mathrm{V}$ & $7.9 \mu \mathrm{V}$ & $1.2 \mu \mathrm{V}$ \\
\hline
\end{tabular}

Table 4 Standard deviation of the recorded sensor voltages

To test how the sensor outputs compare to the theory given in (1), the log of the sensor voltage was plotted against the gap size, and fitted to a straight line. Figure 8 shows the result for old oil, and shows a good fit with the theory. In this case, the number before the exponent is different between $5.74 \mu \mathrm{m}$ and $4.9 \mu \mathrm{m}$, due to the different transmittivity of the filters, different filter bandwidth and different detector sensitivity at the two wavelengths. Figure 9 shows the result for new oil, and shows a less linear relationship, particularly for $5.74 \mu \mathrm{m}$, which are due to the issues with the $3 \mathrm{~mm}$ gap signal mentioned previously. It is also the case that the LambertBeer equation does not hold at higher absorbtions, which may have an effect [12].

According to Figure 2, the absorption at $5.74 \mu \mathrm{m}$ is at least 100 times greater than that at $4.9 \mu \mathrm{m}$ for new oil, which is not reflected in Figure 9 which has the powers of $e$ having a similar magnitude. An explanation for this is that the width of the absorption peak in the IR spectrum is considerably smaller than the bandwidth of the filter, meaning that the 
change in sensor output is smaller than suggested by the theory.

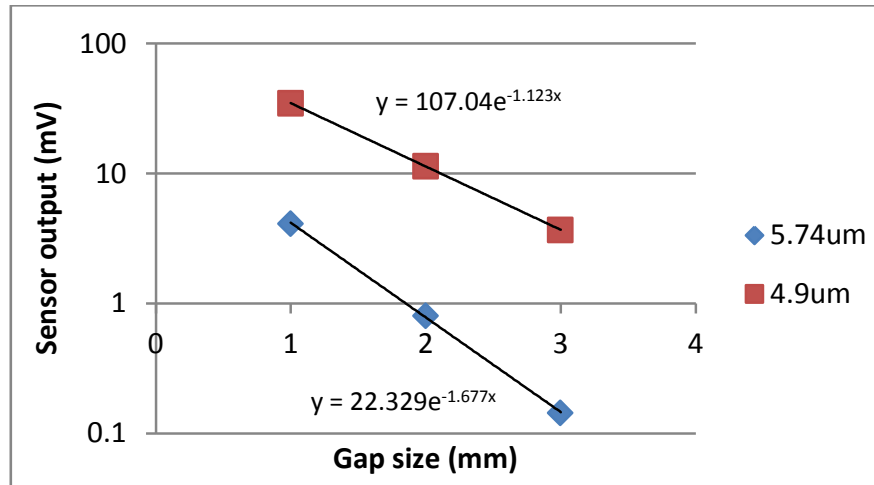

Figure 8 Sensor output vs. gap size, old oil

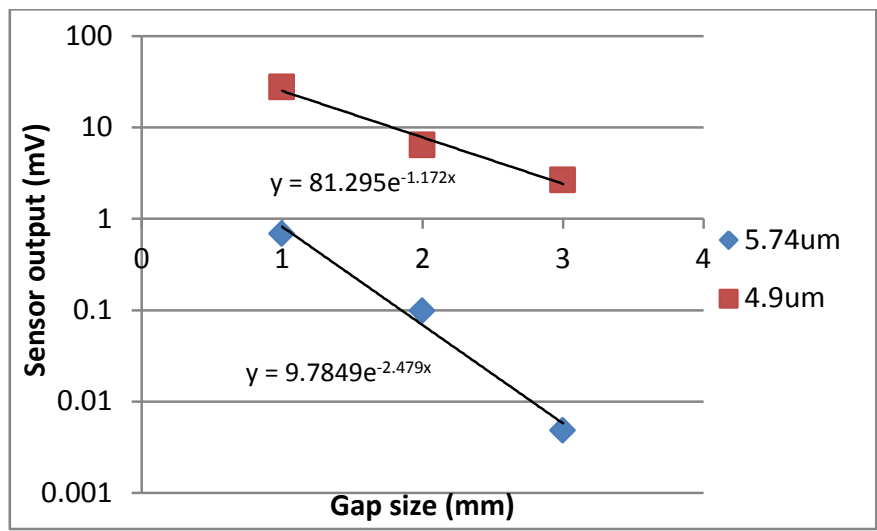

Figure 9 Sensor output vs. gap size, new oil

\section{Conclusion}

Tests have been carried out to discover the feasibility of using NDIR analysis to estimate the degradation of the oil in wind turbine gearboxes, using considerably larger oil gap sizes than previously reported in literature. The tests are based on estimating the level of a depleting additive, which appears as a peak at a wavelength of $5.74 \mu \mathrm{m}$ in the IR spectrum. The results suggest that estimation of the additive depletion should be possible with a $2 \mathrm{~mm}$ gap size or smaller using the signal acquisition and processing techniques described here, but with a $3 \mathrm{~mm}$ gap an anomalously low signal is produced for reasons not known at this stage. To investigate this issue further would require measurements at other gap sizes within the range in this study, which would show whether the $3 \mathrm{~mm}$ result is anomalous or following a trend not predicted by theory.

For a $3 \mathrm{~mm}$ gap size, a good signal is still detectable for used oil, so it may be possible to estimate the additive depletion after some level of depletion has occurred. However, without knowing the initial additive level this may not be accurate. Further work could involve creating oil samples with different additive levels, by mixing old and new oil, to test how the sensors detect the depleting levels.
A significant limitation of this study is that the chemical composition of the oil is not known, and the measured peak is assumed to be an additive which depletes, and the depletion of which would signify the need to replace the oil. It may be the case that the oil is still fully able to perform its function with this additive depleted. Furthermore, the results are specific to the brand of oil used, with other brands likely to contain a different additive package, resulting in different changes to the IR spectrum. Nevertheless, the tests validate the technique of averaging the sensor output over a long time period as a method of allowing a larger gap size in NDIR analysis of oil, which increases the practicality of using NDIR for online analysis.

\section{Acknowledgements}

This work was funded by the ERDF-funded Energy Technology Partnership, in association with Gaia Wind Ltd. The authors would like to thank the ETP and ERDF for allowing this study to be carried out.

\section{References}

[1] A. Agoston, C Schneidhofer, N. Dörr, B. Jakoby. "A concept of an infrared sensor system for oil condition monitoring", Elektroteknik \& Informationstechnik, 125/3, pp. 71-75, (2008).

[2] Axetris Microsystems "Application note - EMIRS200 Supply recommendations and power regulation circuitry", (2009).

[3] T. Bley, E. Pignanelli, A. Schütze. "Multichannel IR Sensor System for Determination of Oil Degradation", $14^{\text {th }}$ Int. Meeting on Chemical Sensors, IMCS, (2012).

[4] Excelitas Technologies. "Infrared Sensing Solutions Catalogue", 2012.

[5] A. Hamilton, F. Quail. "Detailed State of the Art Review for the Different On-Line/In-Line Oil Analysis Techniques in Context of Wind Turbine Gearboxes", ASME 2011 Turbo Expo, pp. 971-988, (2011).

[6] T. Krantz, A. Kahraman. "An Experimental Investigation of the Influence of the Lubricant Viscosity and Additives on Gear Wear", Tribol. Trans. 47, pp. 138-148, (2004).

[7] T. K. Liang, M. Friedrich, D. Lala, K. B. Ozanyan. "Portable Fluorescence Sensor for On-Line Monitoring of Lubricant Oils", Proc. IEEE Sensors, 1 (24-27), pp. 8-11, (2004).

[8] MobilGear ${ }^{\mathrm{TM}}$ XMP, Mobil.com [online] Available at: http://www.mobil.com/Australia-

English/Lubes/PDS/GLXXENINDMOMobilgear_XPM. aspx [accessed: $26^{\text {th }}$ Sep. 2012].

[9] S. Paul, W. Legner, A. Krenkow, G. Müller, T. Lemettais, F. Pradat, D. Hertens. "Chemical Contamination Sensor for Phosphate Ester Hydraulic Fluids", Int. Journal of Aerospace Engineering, 2010, (2010).

[10] I. Poljanšek, M. Krajnc. "Characterization of PhenolFormaldehyde Prepolymer Resins by In Line FTIR 
Spectroscopy", Acta Chimica Slovenica, 52, pp. 238244, (2005).

[11] M. Scherer, M. Arndt, P. Bertrand, B. Jacoby. "Fluid Condition Monitoring Sensors for Diesel Engine Control", Proc. IEEE Sensors, pp. 459-462, (2004).
[12] B.R. Wiesent, D.D. Dorigo, A.W. Koch. "Suitability of tunable Fabry-Perot spectrometers for condition monitoring purposes of gear oils in offshore wind turbines", Proc. IRS ${ }^{2}$ SENSOR+TEST Conferences, (2011). 